\title{
Colonization of long term care facility patients with MDR-Gram-negatives during an Acinetobacter baumannii outbreak
}

Ines Zollner-Schwetz ${ }^{1 *}$ D, Elisabeth Zechner ${ }^{1}$, Elisabeth Ullrich², Josefa Luxner², Christian Pux ${ }^{3}$, Gerald Pichler $^{3}$, Walter Schippinger ${ }^{3}$, Robert Krause ${ }^{1}$ and Eva Leitner ${ }^{2}$

\begin{abstract}
Background: We aimed to determine the prevalence of colonization by multidrug-resistant Gram-negative bacteria including ESBL-producing enterobacteriaceae, carbapenem-resistant enterobacteriaceae, Pseudomonas aeruginosa and Acinetobacter baumannii at two wards caring long term for patients with disorder of consciousness at the Geriatric Health Centers Graz, Austria. During our study we detected two A. baumannii outbreaks.

Methods: In August 2015, we conducted a point-prevalence study. Inguinal and perianal swabs were taken from 38 patients and screened for multidrug-resistant Gram-negative rods using standard procedures. Six months after the initial investigation all patients were sampled again and use of antibiotics during the past 6 months and mortality was registered. Genetic relatedness of bacteria was evaluated by DiversiLab system.

Results: Fifty percent of patients were colonized by multidrug-resistant Gram-negative isolates. Five patients harboured ESBL-producing enterobacteriaceae. No carbapenem-resistant enterobacteriaceae were detected. 13/38 patients were colonized by A. baumannii isolates (resistant to ciprofloxacin but susceptible to carbapenems). There was a significant difference in the prevalence of colonization by A. baumannii between ward 2 and ward 1 ( $60 \%$ vs. $5.6 \%, p<0.001)$. Two clusters of $A$. baumannii isolates were identified including one isolate detected on a chair in a patient's room.

Conclusions: We detected a high prevalence of two multidrug-resistant A. baumannii strains in patients with disorder of consciousness at a LTCF in Graz, Austria. Our findings strongly suggest nosocomial cross-transmission between patients. An active surveillance strategy is warranted to avoid missing newly emerging pathogens.
\end{abstract}

Keywords: Acinetobacter Baumannii, Colonization, Long term care facility, Disorder of consciousness

\section{Background}

Long term care facilities (LTCF) play an essential role in contemporary healthcare systems due to an ageing population in the industrialized world. There is increasing evidence suggesting that residents in LTCFs are frequently colonized by multidrug-resistant Gram-negative bacteria [1-3]. Asymptomatic carriage of multidrug-resistant Gram-negative pathogens constitutes a potential source of transmission to other patients. In addition, there is an

\footnotetext{
* Correspondence: ines.schwetz@medunigraz.at

${ }^{1}$ Department of Internal Medicine, Section of Infectious Diseases and Tropical Medicine, Medical University of Graz, Auenbruggerplatz 15, A-8036 Graz, Austria

Full list of author information is available at the end of the article
}

increased risk of subsequent infection by the multidrug resistant organism [4].

Several organisms are of concern in this setting in particular carbapenemase-producing enterobacteriaceae as well as Acinetobacter baumannii. A. baumannii has been shown to colonize the skin [5] as well as abiotic surfaces like equipment used in ICUs [6]. The ability of A. baumannii to form biofilms is thought to be pivotal for this colonization [6]. Several studies have demonstrated that $A$. baumannii colonizes patients in LTCFs $[3,7,8]$.

The aim of our study was to determine the prevalence of colonization by multidrug-resistant Gram-negative bacteria including ESBL-producing enterobacteriaceae, carbapenem-resistant enterobacteriaceae, $P$. aeruginosa 
und $A$. baumannii at two wards caring long term for patients with disorders of consciousness (unresponsive wakefulness syndrome and minimally conscious state) at the Geriatric Health Centers Graz, Austria. In the course of this study, an outbreak of A. baumannii was discovered and analysed. The results of this analysis are also described in this manuscript.

\section{Methods}

\section{Setting and study design}

We conducted a point-prevalence study in August 2015 at two wards caring long term for patients with disorders of consciousness (unresponsive wakefulness syndrome and minimally conscious state) at the Geriatric Health Centers Graz, Austria. Patients are managed in single or double rooms. The two wards ( 23 beds each) are located in the same building and are staffed by the same team of health care personnel. Two swabs (Copan, Brescia, Italy) were taken from the perianal region and from skin of the inguinal region (pooled from both sides), respectively. Microbiological sampling was repeated 6 months later in February 2016. Healthcare workers were trained how to obtain microbiological swabs. Sampling was scheduled during the morning ward round before bathing and dressing the patients. Informed written consent was obtained from the legal representatives of all patients.

\section{Data collection}

At the initial survey, structured questionnaires were completed for each patient to document demographic and administrative data as well as data concerning possible risk factors for asymptomatic colonization by multidrug-resistant Gram-negative bacteria. Collected variables included: age, gender, length of stay in the facility, bowel and bladder incontinence, previous hospitalisation or surgery (in past 3 months), previous antibiotic use (in the past 3 months), presence of enteral feeding tubes, tracheostomy and/or urinary catheters, and presence of chronic wounds (decubitus, surgical wounds, and chronic vascular ulcers). Questionnaires for the follow-up survey after 6 months included antibiotic treatment in the past 6 months.

\section{Microbiological methods}

Microbiological samples were transported immediately to the microbiological laboratory of the Institute of Hygiene, Microbiology and Environmental Medicine, Medical University of Graz. Swabs were plated on ChromID ESBL, Chrom ID Carba Smart and MacConkey agar plates (bioMerieux, Marcy l'Etoile, France). The plates were incubated under aerobic conditions at $36{ }^{\circ} \mathrm{C}$ and were evaluated for growth after 24 and 48 h. Suspected colonies were further cultivated on blood agar and identified to species level using the automated Vitek MS system (bioMerieux). Antimicrobial susceptibility was tested using Vitek-2 (card AST-N196 and/or N248) with interpretation of the results according to EUCAST breakpoints. All isolates were stored at $-70{ }^{\circ} \mathrm{C}$ for analysis of genetic relatedness at the end of the study. Automated repetitive PCR with the DiversiLab system (bioMérieux, Nürtingen, Germany) was performed to determine clonal relationships following manufacturer's instructions. Isolates with a similarity index $>95 \%$ were considered related and with a similarity index $>97.5 \%$ as indistinguishable.

Multidrug-resistance was defined according to the recommendations of the Robert Koch Institute (RKI) in Germany issued in 2012 [9]. Briefly, isolates resistant to 3 out of 4 relevant antimicrobial classes (acylureidopenicillin, 3rd/4th generation cephalosporins, carbapenems, fluoroquinolones) were classified as 3MRGN. Enterobacteriaceae resistant to carbapenems were classified as 4MRGN even if the isolate remains susceptible to one other antibiotic class. Isolates resistant to all 4 classes were classified as $4 \mathrm{MRGN}$.

\section{Statistical analysis}

Quantitative variables were expressed as mean \pm standard deviation. For statistical analysis Student's t-test, Chi Square test and Fisher's exact test were used as appropriate. A $p$-value of less than 0.05 was considered to indicate statistical significance. The statistical software package SPSS 20.0 (Chicago, IL, USA) was used.

\section{Results}

\section{Patient characteristics}

A total of 46 patients was eligible for the study. 38 patients were included in the study (mean age $58.2 \pm 13.6$ years, 95\% CI: 53.7-62.6 years). Eight patients were not included because of a lack of consent of their legal representatives. 21/38 of included patients were male. The mean duration of stay at the ward was $53.6 \pm 58$ months, $95 \%$ CI: $34.2-$ 72.3 months. All patients had enteral feeding tubes. Three patients had suprapubic urinary catheters. None of the patients had chronic wounds/skin defects. Seventeen patients had tracheostomy. None of the patients required mechanical ventilation. None of the patients had been transferred to an acute care hospital within 3 months prior to the study. Five patients had received antibiotic therapy in the 3 months prior to the study.

\section{Acinetobacter Baumannii outbreak}

$13 / 38$ patients were found to be colonized by 3MRGN A. baumannii isolates. All of these isolates were resistant to ciprofloxacin but susceptible to carbapenems. One patient was co-colonized by an ESBL-producing E. coli isolate and a 3MRGN A. baumannii isolate. In addition, 
5/38 patients were colonized by $A$. baumannii isolates that remained susceptible to carbapenems and ciprofloxacin and were hence not classified as multidrug-resistant. Overall, 18/38 patients were colonized by any A. baumannii isolate. Characteristics of patients colonized by 3 MRGN A. baumannii compared to non-colonized patients are summarized in Table 1. There was a significant difference in the prevalence of colonization by $3 \mathrm{MRGN}$ A. baumannii between ward 2 and ward 1 (60\% vs. 5.6\%, $p<0.001$ ). Patients colonized by 3MRGN A. baumannii had stayed at the ward significantly longer before the study compared to non-colonized patients (91.4 \pm 59 months vs. $33.4 \pm 47.3$ months, $p=0.002)$.

Of 18 patients initially colonized by any A. baumannii isolate 10 were still colonized after 6 months (in February 2016), whereas 6 patients were not colonized any longer (Fig. 1). There was no significant difference in mortality and antibiotic use between patients colonized by MRGN bacteria compared to non-colonized patients.

As 18/38 of patients were colonized by any A. baumannii isolate a source in the environment of ward 2 was suspected. Several studies documenting A. baumannii outbreaks demonstrated that water sources such as sinks and the patients' environment were contaminated by the organism [10-12]. Therefore, swabs were taken from glove boxes, tissue dispensers, sterile filters of a water source, bottles of disinfectant used by cleaning personnel, bedrails, a patient bathtub and a patient elevator into the bathtub, bottles of aromatic oils, sinks in personnel room, a table in personnel room, chairs for visitors in patient rooms. In addition, water drawn from 3 different taps was analysed (source of water for washing patients, kitchen sink, and bathtub). Body care products were not tested as they are used on a single-patient basis. A. baumannii was detected on the patient elevator into bathtub (isolate not available for further analysis) and from a chair for visitors in a patient room. The latter was classified as a 3MRGN A. baumannii and was included in the Diversilab study. In addition, patients' charts were reviewed to identify clinical $A$. baumannii isolates during the study period.

\section{Prevalence of MRGN bacteria}

At the initial survey in August 2015, we detected 19/38 patients harbouring MRGN isolates (overall prevalence $50 \%$, Table 2). Five patients were colonized by 3 MRGN enterobacteriaceae ( 3 by ESBL-producing $E$. coli isolates, 2 by ESBL-producing Klebsiella pneumoniae isolates). No carbapenem-resistant enterobacteriaceae were detected. Two patients were colonized by 4MRGN Pseudomonas aeruginosa isolates.

The follow-up survey was conducted in February 2016. Four patients died during the study period. No swabs were received from 3 patients. Of 5 patients initially colonized by 3MRGN enterobacteriaceae 3 were still colonized, 1 patient was negative, 1 patient had died during the study period. In contrast, 3 patients were newly colonized by 3MRGN enterobacteriaceae at 6 months. Of 2 patients colonized by 4MRGN $P$. aeruginosa at the beginning of the study, one was negative at 6 months and one patient had died. No patient was newly colonized by 4 MRGN $P$. aeruginosa (Table 2). There was no significant difference in mortality and antibiotic use between patients colonized by MRGN bacteria compared to non-colonized patients.

\section{Genetic relatedness}

Twenty-one 3MRGN A. baumannii isolates from patients were included in the DiversiLab analysis as well as the isolate from the environment (chair). One isolate that remained susceptible to ciprofloxacin was also included (patient 9). Two clusters of identical isolates were identified (cluster A and B; Fig. 2). The isolate from the chair was genetically identical to a total of 15 patient isolates and was part of cluster A.

Ten 3MRGN E.coli isolates were included in a separate DiversiLab analysis. Two clusters of identical isolates were detected, comprising 6 and 3 isolates, respectively. One isolate was completely different. At the follow-up

Table 1 Clinical characteristics of 3MRGN A. baumannii colonized vs. non-colonized patients

\begin{tabular}{|c|c|c|c|}
\hline & $\begin{array}{l}\text { Colonized } \\
n=13\end{array}$ & $\begin{array}{l}\text { Non-Colonized } \\
n=25\end{array}$ & $p=$ \\
\hline Age (years, mean $\pm S D$ ) & $54 \pm 17.6$ & $60.4 \pm 10.7$ & 0.257 \\
\hline \multicolumn{4}{|l|}{ Gender (n) } \\
\hline Male & 11 & 10 & \multirow[t]{2}{*}{0.015} \\
\hline Female & 2 & 15 & \\
\hline \multicolumn{4}{|l|}{ Ward (n) } \\
\hline 1 & 1 & 17 & \multirow[t]{2}{*}{$<0.001$} \\
\hline 2 & 12 & 8 & \\
\hline Length of stay (months, mean \pm SD) & $91.4 \pm 59$ & $33.4 \pm 47.3$ & 0.002 \\
\hline Antibiotic therapy past 3 months (n) & 4 & 3 & 0.203 \\
\hline
\end{tabular}




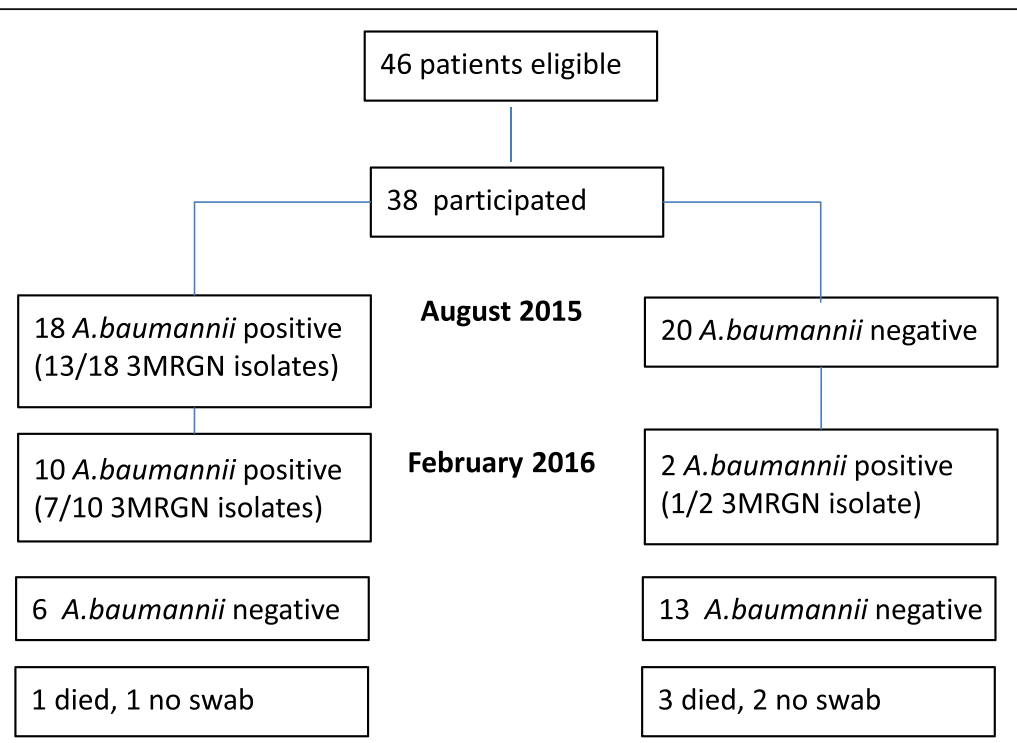

Fig. 1 Number of patients colonized by A. baumannii isolates in August 2015 and February 2016. 3MRGN: Multidrug-resistance was defined according to the recommendations of the Robert Koch Institute (RKI) in Germany issued in 2012 [9]. Isolates resistant to 3 out of 4 relevant antimicrobial classes (acylureidopenicillin, 3rd/4th generation cephalosporins, carbapenems, and fluoroquinolones) were classified as 3MRGN

study (February 2016) 3 patients were found to be newly colonized by 3MRGN E.coli isolates. These isolates were all genetically identical and were part of the cluster of six isolates.

\section{Discussion}

This study analyzed the colonization by multidrugresistant Gram-negative bacteria in patients with disorders of consciousness at a LTCF in Graz, Austria. Notable findings were (1) the prevalence of colonization by multidrug-resistant (3MRGN) A. baumannii was unexpectedly high, in particular among patients in ward 2; (2) two clusters of genetically identical A. baumannii isolates were identified, including one isolate from the environment; (3) colonization by A. baumannii persisted for 6 months in more than half of patients.

A. baumannii has emerged as an important pathogen of healthcare-associated infections in critically ill patients in the ICU setting worldwide [10, 13, 14]. In addition, several studies documented that $A$. baumannii

Table 2 Prevalence of colonization by MRGN bacteria

\begin{tabular}{|c|c|c|}
\hline & August 2015 & February 2016 \\
\hline & Number of patients & Number of patients \\
\hline E. coli (3MRGN) & $3^{a}$ & $6^{b}$ \\
\hline K. pneumoniae (3MRGN) & 2 & 0 \\
\hline P. aeruginosa (4MRGN) & 2 & 0 \\
\hline A. baumannii (3MRGN) & $13^{a}$ & 7 \\
\hline
\end{tabular}

also occurs in patients in LTCFs [3, 7, 8]. In Maryland Thom and colleagues investigated the colonization of patients by $A$. baumannii in LTCFs providing care to mechanically ventilated patients [15]. The authors demonstrated that $63 \%$ of patients were colonized by A. baumannii. This is comparable to our findings although none of the patients in our study required mechanical ventilation. The high prevalence of colonization by $A$. baumannii could be explained by the fact that $90 \%$ of patients are transferred to the LCTF directly from external ICUs. In 2015, one fourth of the newly admitted patients were colonized by a multidrug-resistant pathogen at the time of admission. Although all newly admitted patients are screened for multidrug-resistant pathogens, skin sites were until now only screened for the presence of methicillin-resistant Staphylococcus aureus. Screening of the skin for multidrug-resistant Gram-negative rods has been introduced as a consequence of our study (see below).

The prevalence of colonization by A. baumannii was unexpectedly high in our study with an accumulation of cases in ward 2. We therefore decided to explore the environment and water sources of this ward to identify a source. A. baumannii isolates were detected on the patient elevator into a bathtub (isolate not available for further analysis) and from a chair for visitors in a patient room. The latter isolate was genetically identical to a total of 15 patient isolates. In addition, patients colonized by A. baumannii had stayed at the ward significantly longer before the study compared to noncolonized patients. Taken together, these findings 


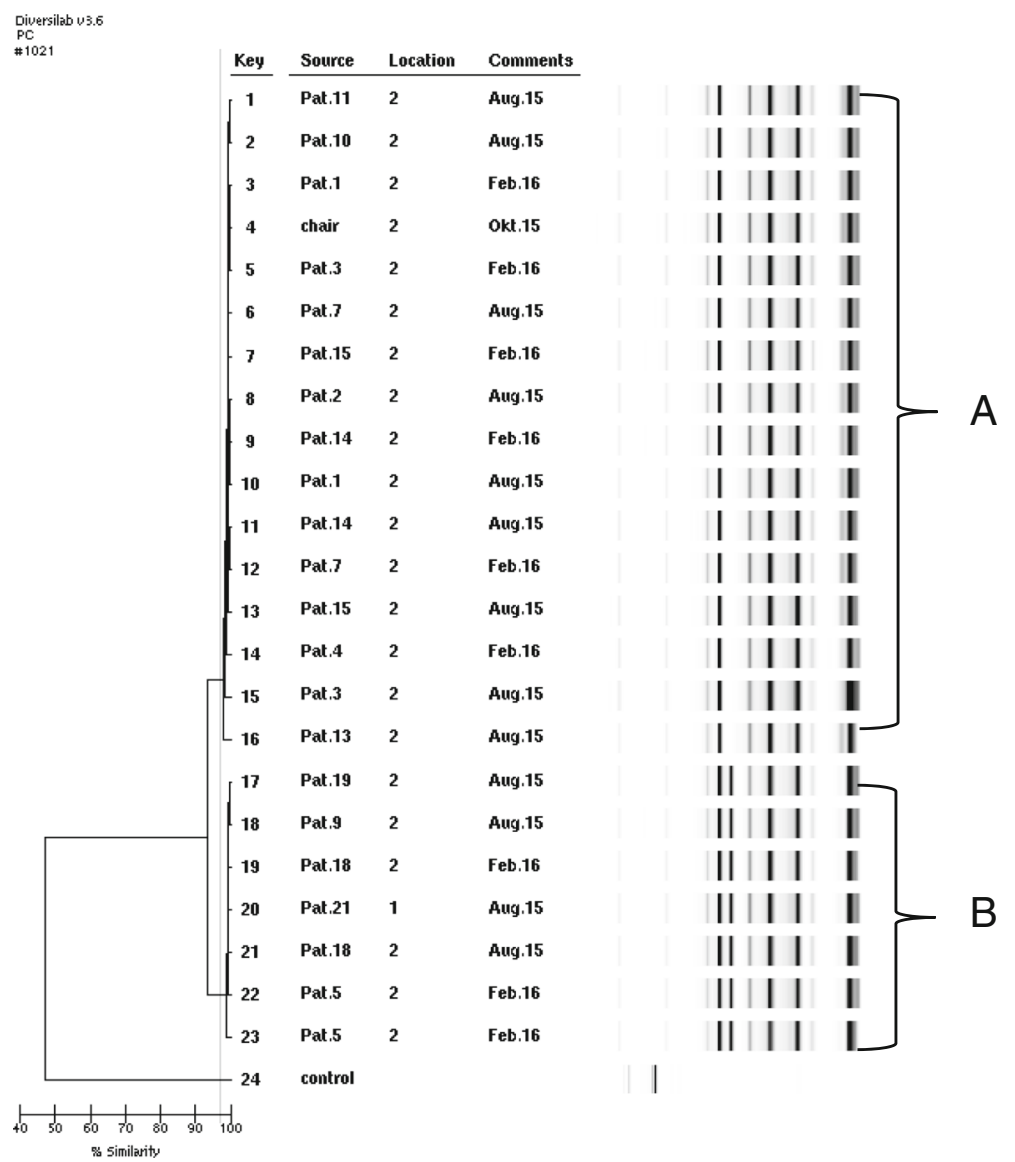

Fig. 2 Genetic relatedness of $23 \mathrm{~A}$. baumannii isolates by Diversilab System Isolates with a similarity index $>95 \%$ were considered related and with a similarity index $>97.5 \%$ as indistinguishable. The grey line indicates $97.5 \%$ similarity. Location: ward 1 or ward 2 . Two clusters of A. baumannii isolates were detected (cluster A and cluster B)

suggest that cross-transmission between patients by staff may have taken place.

As a consequence of our findings a multimodal intervention program was introduced on both wards by the infection control staff at the end of the study period. It included consequent reinforcement of standard hygiene precautions and barrier precautions as well as repeated education for all occupational groups also including visitors. Colonized patients were washed with antiseptic soap once a month. In addition, disinfection protocols were reviewed specifically addressing the patients' environment (including the bathtub and the elevator). The time frame for cleaning personnel on both wards was increased by $1 \mathrm{~h}$ per day. Admission screening procedures for new patients were adapted to include multidrugresistant Gram-negative bacteria also from skin swabs. All patients on both wards will be screened for multidrug-resistant bacteria twice a year. These measures were evaluated in a follow-up survey by the infection control staff in December 2016. Only 4/22 patients (18\%) in ward 2 were still colonized by 3MRGN
A. baumannii. The long-term effects of these measures will be evaluated by a follow-up study.

Of note A. baumannii was identified from a clinical sample only once during the entire study period, indicating that our patients were in fact only colonized but not infected by $A$. baumannii. Using only clinical samples for surveillance purposes would have underestimated the true prevalence of multidrug-resistant organisms in our setting. Our findings favour the implementation of an active surveillance strategy.

In contrast to the high prevalence of colonization by A. baumannii the prevalence of multidrug-resistant enterobacteriaceae was moderate (13\%). Our findings are comparable to a French study which reported $10 \%$ of nursing home residents to be colonized by ESBLproducing enterobacteriaceae [16]. In contrast to an Italian study, in which colonization by carbapenemaseproducing enterobacteriaceae has been described in 6.3\% of patients, no carbapenemase-producing enterobacteriaceae were detected [1]. Sixty percent of patients were still colonized by 3MRGN E.coli isolates at the time of 
the follow-up study. Our findings are in line with a study by Birgand et al. investigating the gastrointestinal ESBLcolonization in patients after hospital discharge [17]. Median time to clearance was found to be 6.6 months, ranging from 3.4 to 13.4 months [17]. Three patients were found to be newly colonized by 3MRGN E.coli isolates at the time of the follow-up study. These 3 isolates were found to be genetically identical, again pointing to a role of institutional cross-transmission in the spread of multidrug resistant bacteria.

Our study has limitations. To fully assess the prevalence of colonization by $A$. baumannii respiratory samples would probably have been a useful addition. However, our study was designed to assess the prevalence of multidrugresistant Gram-negative pathogens in general. The high prevalence of $A$. baumannii was unexpected.

\section{Conclusion}

We uncovered two clusters of $A$. baumannii colonizing patients with disorder of consciousness at a LTCF in Graz, Austria. Our findings point toward nosocomial crosstransmission as a cause for these outbreaks. Using only clinical samples for surveillance purposes would have underestimated the true prevalence of $A$. baumannii in our setting. Therefore, an active surveillance strategy is warranted to avoid missing newly emerging pathogens at an early stage. In addition, improved infection control measures are necessary in the LTCF setting.

\section{Abbreviations \\ 3MRGN: multidrug resistant Gram-negative bacteria according to the defin- ition of Robert Koch Institute; ESBL: extended spectrum betalactamases; EUCAST: European Committee on Antimicrobial Susceptibility Testing; ICU: intensive care unit; LTCF: long term care facility}

\section{Acknowledgments}

Not applicable.

Funding

No external funding was received for this study.

\section{Availability of data and materials}

The datasets analysed during the current study are available from the corresponding author on reasonable request.

\section{Authors' contributions}

$E Z, E U$ and EL processed the swabs and analysed the microbiological data. $J L$ performed DiversiLab analysis and contributed to the manuscript. CP, GP and WS planned the study and contributed to the manuscript. IZS and RK analysed the data and wrote the manuscript. All authors read and approved the final manuscript.

\section{Competing interests}

The authors declare that they have no competing interests.

\section{Consent for publication}

Not applicable.

\section{Ethics approval and consent to participate}

Informed written consent was obtained from the legal representatives of all patients. The study was approved by the local ethics committee (votum number: 27-378 ex 14/15 Medical University of Graz).

\section{Publisher's note}

Springer Nature remains neutral with regard to jurisdictional claims in published maps and institutional affiliations.

\section{Author details}

'Department of Internal Medicine, Section of Infectious Diseases and Tropical Medicine, Medical University of Graz, Auenbruggerplatz 15, A-8036 Graz, Austria. ${ }^{2}$ Institute of Hygiene, Microbiology and Environmental Medicine, Medical University of Graz, Graz, Austria. ${ }^{3}$ Geriatric Health Centers of the City of Graz, Graz, Austria.

Received: 31 January 2017 Accepted: 11 May 2017

Published online: 16 May 2017

\section{References}

1. March A, Aschbacher R, Dhanji H, Livermore DM, Bottcher A, Sleghel F, Maggi S, Noale M, Larcher C, Woodford N. Colonization of residents and staff of a long-term-care facility and adjacent acute-care hospital geriatric unit by multiresistant bacteria. Clin Microbiol Infect. 2010;16:934-44.

2. Furuno JP, Hebden JN, Standiford HC, Perencevich EN, Miller RR, Moore AC, Strauss SM, Harris AD. Prevalence of methicillin-resistant Staphylococcus aureus and Acinetobacter Baumannii in a long-term acute care facility. Am J Infect Control. 2008;36:468-71.

3. Lim CJ, Cheng AC, Kennon J, Spelman D, Hale D, Melican G, Sidjabat HE, Paterson DL, Kong DC, Peleg AY. Prevalence of multidrug-resistant organisms and risk factors for carriage in long-term care facilities: a nested case-control study. J Antimicrob Chemother. 2014;69:1972-80.

4. Reddy P, Malczynski M, Obias A, Reiner S, Jin N, Huang J, Noskin GA, Zembower T. Screening for extended-spectrum beta-lactamase-producing Enterobacteriaceae among high-risk patients and rates of subsequent bacteremia. Clin Infect Dis. 2007;45:846-52.

5. Sebeny PJ, Riddle MS, Petersen K. Acinetobacter Baumannii skin and softtissue infection associated with war trauma. Clin Infect Dis. 2008;47:444-9.

6. Gaddy JA, Actis LA. Regulation of Acinetobacter Baumannii biofilm formation. Future Microbiol. 2009:4:273-8.

7. Sengstock DM, Thyagarajan R, Apalara J, Mira A, Chopra T, Kaye KS. Multidrug-resistant Acinetobacter Baumannii: an emerging pathogen among older adults in community hospitals and nursing homes. Clin Infect Dis. 2010;50:1611-6.

8. Mortensen E, Trivedi KK, Rosenberg J, Cody SH, Long J, Jensen BJ, Vugia DJ. Multidrug-resistant Acinetobacter Baumannii infection, colonization, and transmission related to a long-term care facility providing subacute care. Infect Control Hosp Epidemiol. 2014;35:406-11.

9. Hygienemaßnahmen bei Infektionen oder Besiedlung mit multiresistenten gramnegativen Stäbchen. Bundesgesundheitsblatt: Springer; 2012;55:1311-1354.

10. Wang SH, Sheng WH, Chang YY, Wang LH, Lin HC, Chen ML, Pan HJ, Ko WJ, Chang SC, Lin FY. Healthcare-associated outbreak due to pan-drug resistant Acinetobacter Baumannii in a surgical intensive care unit. J Hosp Infect. 2003:53:97-102.

11. Umezawa K, Asai S, Ohshima T, Iwashita H, Ohashi M, Sasaki M, Kaneko A, Inokuchi S, Miyachi H. Outbreak of drug-resistant Acinetobacter Baumannii ST219 caused by oral care using tap water from contaminated hand hygiene sinks as a reservoir. Am J Infect Control. 2015;43:1249-51.

12. Hong KB, Oh HS, Song JS, Lim JH, Kang DK, Son IS, Park JD, Kim EC, Lee HJ, Choi EH. Investigation and control of an outbreak of imipenem-resistant Acinetobacter Baumannii infection in a pediatric intensive care unit. Pediatr Infect Dis J. 2012;31:685-90.

13. Enoch DA, Summers C, Brown NM, Moore L, Gillham MI, Burnstein RM, Thaxter R, Enoch LM, Matta B, Sule O. Investigation and management of an outbreak of multidrug-carbapenem-resistant Acinetobacter Baumannii in Cambridge, UK. J Hosp Infect. 2008;70:109-18.

14. Frickmann H, Crusius S, Walter U, Podbielski A. Management of an Outbreak with cases of Nosocomial pneumonia caused by a novel multi-drugresistant Acinetobacter Baumannii clone. Pneumologie. 2010;64:686-93.

15. Thom KA, Maragakis LL, Richards K, Johnson JK, Roup B, Lawson P, Harris AD, Fuss EP, Pass MA, Blythe $D$, et al. Assessing the burden of Acinetobacter Baumannii in Maryland: a statewide cross-sectional period prevalence survey. Infect Control Hosp Epidemiol. 2012;33:883-8.

16. Cochard H, Aubier B, Quentin R, van der Mee-Marquet N, Reseau des Hygienistes du C. Extended-spectrum beta-lactamase-producing 
Enterobacteriaceae in French nursing homes: an association between high carriage rate among residents, environmental contamination, poor conformity with good hygiene practice, and putative resident-to-resident transmission. Infect Control Hosp Epidemiol. 2014;35:384-9.

17. Birgand G, Armand-Lefevre L, Lolom I, Ruppe E, Andremont A, Lucet JC. Duration of colonization by extended-spectrum beta-lactamaseproducing Enterobacteriaceae after hospital discharge. Am J Infect Control. 2013;41:443-7.

Submit your next manuscript to BioMed Central and we will help you at every step:

- We accept pre-submission inquiries

- Our selector tool helps you to find the most relevant journal

- We provide round the clock customer support

- Convenient online submission

- Thorough peer review

- Inclusion in PubMed and all major indexing services

- Maximum visibility for your research

Submit your manuscript at www.biomedcentral.com/submit 